\title{
Enhancement of integrated photonic biosensing by magnetic controlled nano-particles
}

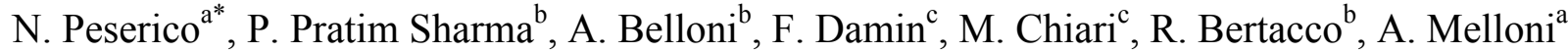 \\ ${ }^{a}$ Dip. Elettronica, Informatica e Bioingegneria, Politecnico di Milano, via Colombo 81, Milan, Italy; \\ ${ }^{\mathrm{b}}$ Dip. Fisica, Politecnico di Milano, via Colombo 81, Milan, Italy; ${ }^{\mathrm{c}}$ ICRM-CNR, via Mario Bianco \\ 9, Milano, Italy.
}

\begin{abstract}
Integrated Mach-Zehnder interferometers, ring resonators, Bragg reflectors or simple waveguides are commonly used as photonic biosensing elements. They can be used for label-free detection relating the changes in the optical signal in realtime, as optical power or spectral response, to the presence and even the quantity of a target analyte on the surface of the photonic waveguide. The label-free method has advantages in term of sample preparation but it is more sensitive to spurious effects such as temperature and refractive index sample variation, biological noise, etc. Label methods can be more robust, more sensitive and able to manipulate the biological targets.

In this work, we present an innovative labeled biosensing technique exploiting magnetic nano-beads for enhancement of sensitivity over integrated optic microrings. A sandwich binding is exploited to bring the magnetic labels close to the surface of the optical waveguide and interact with the optical evanescent field.

The proximity and the quantity of the magnetic nano-beads are seen as a shift in the resonance of the microring. Detection of antibodies permits to reach a high level of sensitivity, down to $8 \mathrm{pM}$ with a high confidence level. The sizes of the nano-beads are 50 to $250 \mathrm{~nm}$. Furthermore, time-varying magnetic fields permit to manipulate the beads and even induce specific signals on the detected light to easy the processing and provide a reliable identification of the presence of the desired analyte. Multiple analytes detection is also possible.
\end{abstract}

Keywords: Biosensors, nanoparticles, label-based, magnetism

\section{INTRODUCTION}

The last decade has seen an increasing interest in biosensing, responding to an increasing request to have new sensible and fast biochemical analyses from hospitals, clinics, and public institutions. New devices, based on different nanotechnologies, have been proposed in the literature and on the market, capable to reach new levels in terms of Limitof-Detection or analytes multiplexing. Exploitation of technologies such as bioFET [1], magnetic tunnel junctions [2], microfluidics [3], etc, has shown exceptional results. Detection of proteins, complementary strain of DNA, virus, bacteria, and more are daily goals, reaching lower and lower concentration, down to pM level or less.

Out of the many technologies, the optical approach has shown significant results in the field [4]. Detection of biological molecules using optical field exploits the effects that such molecules can induce to the propagating light. Most of the light characteristics are subject to changes induced by biochemical interaction, in terms of phase, losses, polarization, and so on. In particular, optical biosensors based on the refractive index changes detection are one of the most explored fields, mainly due to the possibility to integrate these schemes into an integrated optical circuit. Mach-Zehnder interferometers, ring resonators, Bragg reflectors or simple waveguides are commonly used as integrated optical biosensing elements. In particular, microring resonators whose resonance wavelength depends on the effective index of the propagating light inside the ring, show important results when used as biosensors [5].

As the counterpart of the transducer side of a biosensor, an essential role in the definition of the sensor characteristics is done by the biolayer. This layer is the one in charge of capturing the target analytes while avoiding non-specific binding that would create false-positive results. This task is performed after its functionalization with ad-hoc receptors. There are several working schemes regarding this layer. We can divide them into two main categories: label-free and label-based

*nicola.peserico@polimi.it; phone+39022399 8979; http://photonics.deib.polimi.it 
schemes. The later one is the first scheme adopted in biosensing since the early '50, where target analytes are linked with radioactive molecules, detectable by ad-hoc instrumentation. This scheme is also used in the most spread biosensing test, the ELISA test, where fluorescence labels are used [6]. However, in the last decade, label-free biosensors started to appear in literature and on the market, promising low concentration detection together with fast response. In this scheme, the transducer layer has to detect directly the binding between receptors and analytes, without any added molecules. Since the detection is performed in just a single step, the measure is more rapid than label-based ones. However, this scheme tends to inherit some issues, such as false-positive results due to sample variation, and not due to binding events.

In this paper, we present a scheme that mixes optical transducer with a label-base approach. While the optical part uses an integrated microring resonator, a well-known component for many biosensors, we use magnetic beads as labels. These labels have several advantages: first of all, the superparamagnetic core of the beads has a large impact on the propagating mode of the light into the waveguide. Another aspect is the various dimensions that the labels can have, that permit to choose the best one for the target molecules. Moreover, the labels add another binding step to the process of molecule detection, lowering the possibilities of having false-positive results. These advantages allow testing a working biosensor capable of detecting IgG antibodies down to sub-pM concentration, 3 orders of magnitude better than equal label-free biosensor [5]. Furthermore, we exploit the magnetic core of the beads in a novel opto-magnetic platform, where beads are moved toward the optical sensor by an externally driven electromagnet, producing cyclic signal detected by the optical transducer. This platform permits to have an "on-off" signal as the output of the sensor, increasing the robustness of the sensing due to the multi-level condition that produces the signal.

The paper is divided into 4 sections: the second section shows the optical circuit, the magnetic beads, and the biological receptors and analytes used. Experimental results showing the enhancement of the resonance shifts are presented in the third section. The last section is dedicated to the opto-magnetic platform, its working principles, and some preliminary results.

\section{METHODS}

To prove the enhancement of the detection using magnetic beads as labels, we detected IgG antibodies binding over microring resonator. The protocol is formed by three steps, shown in figure 1a-c, summarized in the functionalization of the surface with IgG receptors, binding with complementary antibodies and, by last, binding of target analytes with magnetic labels. During the last 2 steps, wavelength position of the microring resonance is monitored in order to detect binding shifts, as shown in figure $1 \mathrm{~d}$.

a)

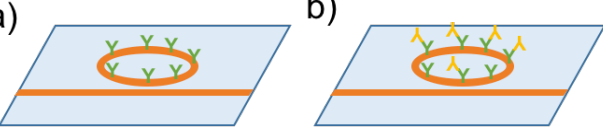

c)

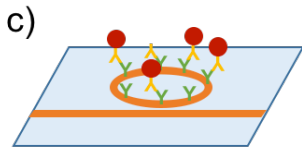

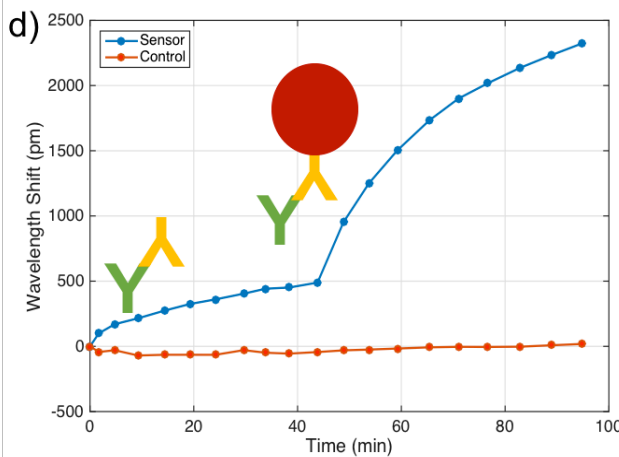

Figure 1: Representations of the experimental steps and results. a-c) Steps of the binding protocol, starting from the functionalization, the binding with target analyte, and the binding with labels. d) Wavelength shifts of microring sensor and control while binding with analyte and labels. The red curve is the control ring response.

\subsection{Optical Chip}

The optical chip is formed by a Silicon Nitride waveguide, whose cross-section dimensions are $1200 \mathrm{~nm}$ wide and 300 $\mathrm{nm}$ thick. The waveguide is placed over a $\mathrm{SiO}_{2}$ layer, while the coupling from the external laser and photodetector is performed by grating couplers with $1550 \mathrm{~nm}$ central wavelength. The microring has $100 \mu \mathrm{m}$ radius, that corresponds to 
an FSR of $2 \mathrm{~nm}$ at $1550 \mathrm{~nm}$ wavelength. The waveguide of the microring resonator is not covered by any cladding, so it can be put in contact with the solution containing the analytes. The monitoring of the resonance position is performed by a tunable laser continuous sweep over a FSR. A similar microring without any functionalization is monitored as control sensor to take into account any source noises not due to binding activity.

\subsection{Functionalization and Binding}

A copolymer provides the base for the functionalization, in particular, we used the copoly(DMA-NAS-MAPS) [7]. The three components of the copolymer are essentials, due to the different functions each one has. Briefly, the DMA is the backbone of the polymer and gives the main bind to the surface. NAS is the reactive group able to bind to DNA and proteins. MAPS, by last, stabilizes the coating. This functionalization binds antibodies to an active conformation so that they preserve their functional activity.

The receptors are then deposited by a micro-spotter, using 1 nanoliter of a solution containing $1 \mu \mathrm{g} / \mathrm{ml}$ of Sigma-Aldrich IgG from rabbit serum (product code: I5006). The spot functionalizes an area that is of the same size of the microring. The complementary binding is made with a solution containing different concentrations of Jackson ImmunoResearch Biotin- SP-AffiniPure Goat Anti-Rabbit $1 \mathrm{IgG}(\mathrm{H}+\mathrm{L})$ (product code: JI111065003). The biotin that is linked to these complementary antibodies is used for binding to the magnetic beads. The IgG-IgG binds are performed in Petri dishes, by immersing the chip for 50 minutes in a controlled atmosphere (with $50 \%$ of humidity).

Magnetic beads are purchased from Micromode. We tested two sizes of beads: the nanomag-D-spio 1 streptavidin $50 \mathrm{~nm}$ (product code: 79-19- 501) and the nanomag-D 1 streptavidin, $130 \mathrm{~nm}$ (product code: 09-19-132). For each one, the binding is performed directly in the measurement setup, on the optical chip surface, monitoring in real time the shift of the microrings. A solution containing magnetic beads is prepared and flow over the chip. Typical measurement lasts 50 minutes, where beads solution completely covers the sensing part of the chip. In these first experiments, no magnetic field is used to accelerate the binding that occurs just by beads diffusion in the solution.

\section{EXPERIMENTAL RESULTS}

In this section, we present the experimental results obtained with our method. The first part shows the difference between the two beads dimensions in terms of optical behavior of the microring. The second part shows the calibration curve of the system, using the larger size of beads.

\subsection{Beads enhancement of the wavelength shift}

After binding two equal microrings with $81 \mathrm{nM}$ target $\mathrm{IgG}$ analytes, we inject $50 \mathrm{~nm}$ beads on the first ring and $130 \mathrm{~nm}$ beads on the second one. For both, we monitored the evolution of the microring optical spectrum in terms of wavelength shift and extinction ratio (ER). Results are plotted in figure 2 . The trend for both is similar, having a red shift in wavelength and a reduction of the ER. In particular, the experiments show significant higher changes using larger beads. Considering the same time window, the $50 \mathrm{~nm}$ beads show $450 \mathrm{pm}$ wavelength shift, while $130 \mathrm{~nm}$ beads produce almost $1250 \mathrm{pm}$ shift. The reduction of the ER can be attributed to the increasing losses per turn that beads induce to the optical light propagating inside the cavities. 

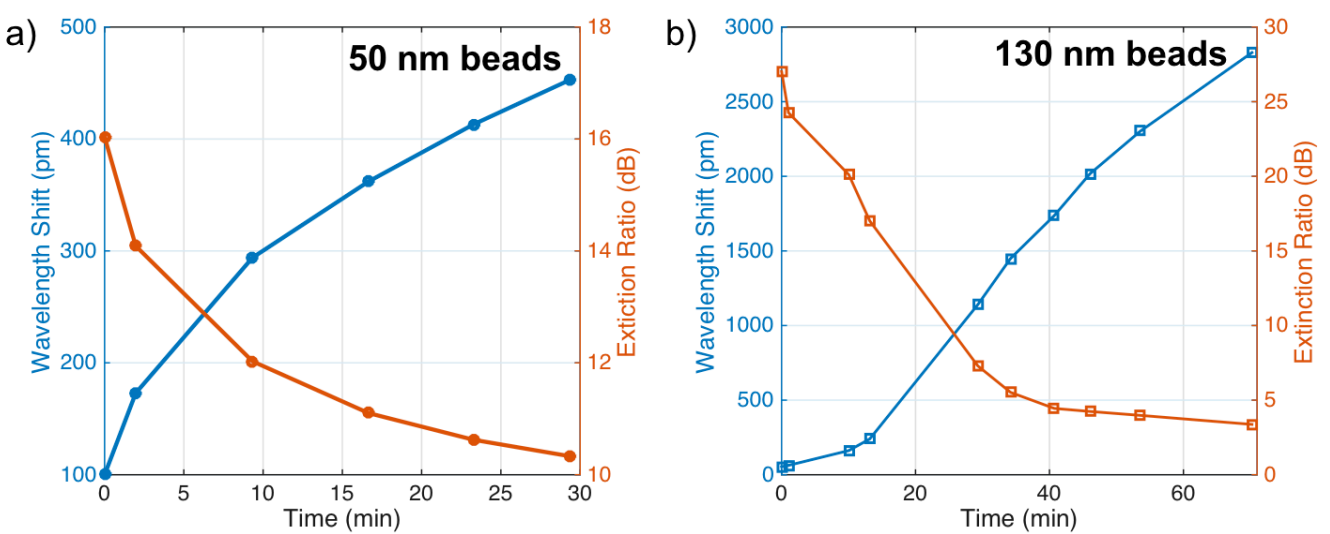

Figure 2: Wavelength shifts and ER changes during binding experiment between target analyte and magnetic beads, for different dimension of the beads: a) $50 \mathrm{~nm}$ beads, b) $130 \mathrm{~nm}$ beads.

\subsection{Calibration curve and Limit-of-Detection}

Considering the 2 dimensions, we selected the larger one to conduct more experiments and fully characterize our biosensor platform. Different chips are bound with several concentrations of the target analyte, from $810 \mathrm{fM}$ to $81 \mathrm{nM}$. After the binding between receptors and analyte, solutions containing the same number of beads are flowed over the sensing area, while measurements of microrings shift are performed. Results of shift for concentration range $8.1 \mathrm{pM}$ to $81 \mathrm{nM}$ are plotted in figure $3 \mathrm{a}$.

a)

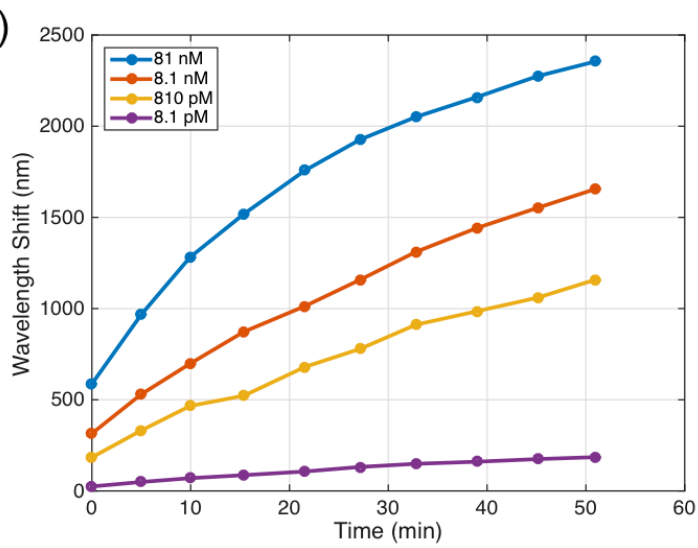

b)

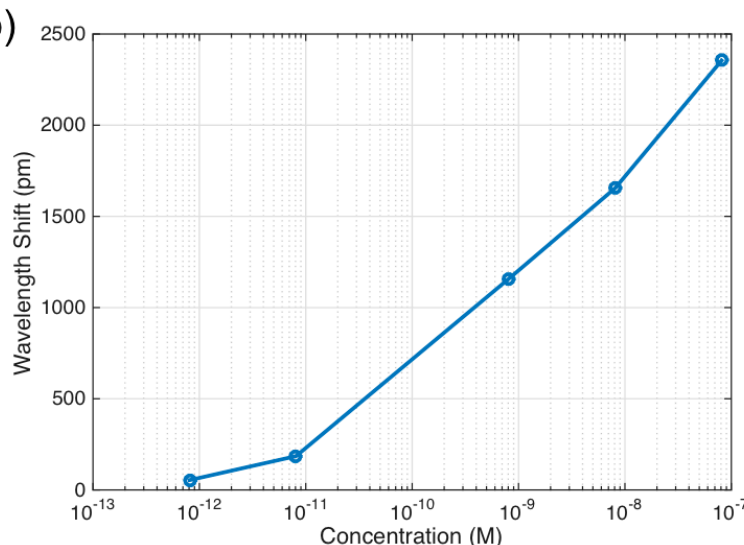

Figure 3: Response of the ring at different concentration and calibration curve. a) Wavelength shifts induced by the binding of the beads on different analyte concentration over the microring resonator. b) A calibration curve, considering final value of wavelength shifts. The biosensor is capable to detect analytes down to $810 \mathrm{fM}$.

The calibration curve is presented in figure $3 \mathrm{~b}$. The biosensor shows an exponential trend from $8 \mathrm{pM}$ to $81 \mathrm{nM}$, meaning a full 4 decades of sensing range. Moreover, the biosensor is capable also to reach a Limit-of-Detection of $810 \mathrm{fM}$. This result is significant since it outperforms similar label-free biosensors results [5]. However, false-positive results could always appear also in this protocol. Moreover, since wavelength shifts for high concentration tend to be quantitatively similar to the Free Spectrum Range of the microring, real-time monitoring of wavelength shift is mandatory. 


\section{OPTO-MAGNETIC BIOSENSING PLATFORM}

To overcome these limitations, we exploit the magnetic core of the beads by applying a sinusoidal magnetic field and detecting the displacement induced to the beads by monitoring microring resonance shift. The scheme and the principle are shown in figure $4 \mathrm{a}-\mathrm{d}$.

a)

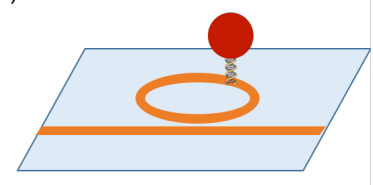

c)

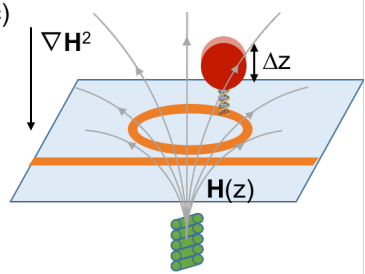

b)

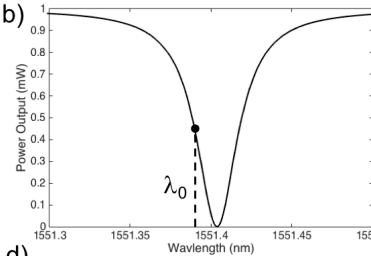

d)

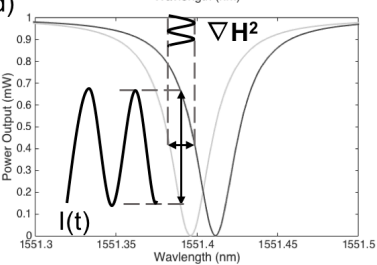

e)

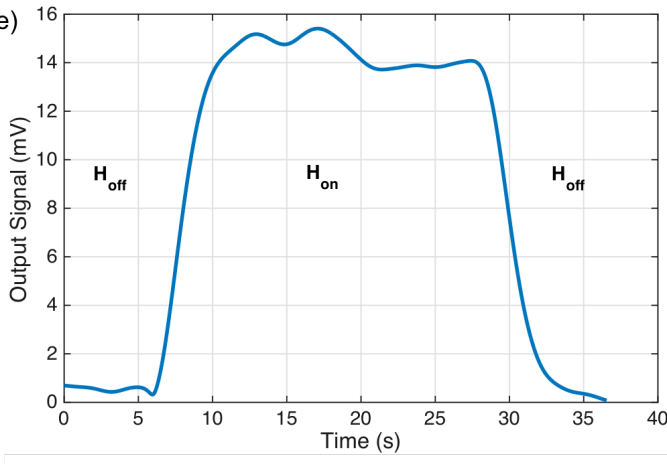

Figure 4: Working principle and output signal of the opto-magnetic biosensing platform. a-b) Initial platform situation, where beads are over the microring and laser is set at the slope of the optical notch. c-d) By applying a magnetic field, the beads are displaced toward the microring, by so shifting the resonance position. e) Demodulated output signal at first harmonic shows the signal appears only when magnetic field is active.

An oscillating sinusoidal current at frequency $\mathrm{f}_{0}$ flowing into an electromagnet placed under the chip generates the magnetic field, whose squared gradient $\nabla \mathbf{H}^{2}$ induces a periodical attractive force on the magnetic beads at frequency $2 * \mathrm{f}_{0}$. The beads react by moving toward the waveguide by a small distance $\Delta \mathrm{z}$ and thus inducing a small change in the effective index of the propagating light inside the cavity. Using a fixed $\lambda_{0}$ wavelength laser placed in the slope of the optical notch, it is possible to detect the signal induced by the beads, in our case, using a lock-in amplifier after collecting output photodiode signal. The output signal $\mathrm{I}(\mathrm{t})$, demodulated at the first harmonic $2 * \mathrm{f}_{0}$, is plotted in figure $4 \mathrm{e}$. The amplitude of the output signal is proportional to the number of bound beads, that is determined by the concentration of the analyte. It is important to highlight that the signal appears only in the presence of magnetic beads, magnetic field, and biological links. The possibility of false-positive results drastically decreases, while the null interference between optical and magnetic field ensure the correct detection. Moreover, the detection is possible when the magnetic field is applied, resulting in an "on-off" measurement. In the case of figure 4e, we use 60-base double-strand DNA as biological probes, at a concentration of $1 \mu \mathrm{M}$. The long DNA permits to have a larger bending of the structure that is mandatory for the displacement of the beads.

\section{CONCLUSION}

In this paper, we showed the possibilities of a synergy of integrated optics and magnetic beads in the field of biosensing. While the first part is dedicated only on the enhancement of the optical biosensing performances using beads, reaching sub-pM concentration, the second part is dedicated to a novel opto-magnetic platform for a more robust detection of biological molecules. These preliminary results show significant features for the future biosensing platform, considering also analytes multiplexing or, considering that on-off measurements can be performed in different places, also sensing in harsh environment. Moreover, system that enables better resolution on the resonance shift detection can improve the overall platform [8]. The future steps will focus on the demonstration of the capabilities of the opto-magnetic platform, exploiting all the possibilities that the flexibility of optical and magnetic fields have. 


\section{REFERENCES}

[1] Schöning, M.J. and Poghossian, A. "Recent advances in biologically sensitive field-effect transistors (biofets). " Analyst, 127(9):1137-1151, (2002).

[2] Sharma, P.P., Albisetti, E., Massetti, M., Scolari, M., La Torre, C., Monticelli, M., Leone, M., Damin, F., Gervasoni, G., Ferrari, G. and Salice, F. "Integrated platform for detecting pathogenic dna via magnetic tunneling junction-based biosensors. " Sensors and Actuators B: Chemical, 242:280-287, (2017).

[3] Balagaddé, F.K., You, L., Hansen, C.L., Arnold, F.H. and Quake, S.R. "Long-term monitoring of bacteria undergoing programmed population control in a microchemostat. " Science, 309(5731):137-140, (2005).

[4] Estevez, M.C., Alvarez, M. and Lechuga, L.M. "Integrated optical devices for lab-on-a-chip biosensing applications." Laser \& Photonics Reviews, 6(4):463-487, (2012).

[5] Peserico, N., Annoni, A., Varriale, A., D'Auria, S., Bellieres, L., Cuesta-Soto, F., Rodrigo, M., Peransi, S. and Melloni, A. "Experimental demonstration of integrated photonic free-label biosensor for CBRN threats using micro-ring resonators." In Transparent Optical Networks (ICTON), 2016 18th International Conference on, pp. 1-4. IEEE, (2016).

[6] Leng, S.X., McElhaney, J.E., Walston, J.D., Xie, D., Fedarko, N.S. and Kuchel, G.A. "Elisa and multiplex technologies for cytokine measurement in inflammation and aging research." The Journals of Gerontology Series A: Biological Sciences and Medical Sciences, 63(8):879- 884, (2008).

[7] Cretich, M., Pirri, G., Damin, F., Solinas, I. and Chiari, M. "A new polymeric coating for protein microarrays." Analytical biochemistry, 332(1):67-74, (2004).

[8] Peserico, N., Barbi, P., Bruccoleri, M., Baudot, C., Fincato, A., Zuffada, M., Guardiani, C. and Melloni, A. "System for tracking femtometer resonance shifts of silicon photonics microring resonator by locking tunable laser", SPIE Photonic West, (2018). 